\title{
On the Topography of Electron Momentum Densities of Linear Molecules*
}

\author{
Sudhir A. Kulkarni ${ }^{1}$ and Shridhar R. Gadre ${ }^{1,2}$ \\ 1 Department of Chemistry, University of Poona, Pune-411 007, Maharashtra, India \\ 2 Institut für Physikalische Chemie und Elektrochemie der Universität Karlsruhe, Karlsruhe, FRG
}

Z. Naturforsch. 48a, 145-150 (1993); received January 3, 1992

\begin{abstract}
A topographical study of the electron momentum density (EMD) of some linear molecules is presented with special emphasis on the bond-directionality principle. A new approach to the bonddirectionality (BD) principle has been proposed. This is based on the analysis of curvatures of critical points at $p=0$ and elsewhere on the bonding axis. The linear molecules are classified into three broad categories: those which fully obey or disobey the BD principle as well as those which satisfy it only partly. The Laplacian of the EMD at $p=0$ has been associated with the quality of the wavefunction via the electron density in the tail region. Also, the similarity in the critical structure of both the EMD and the spherically averaged EMD at the origin is brought out.
\end{abstract}

Key words: Electron momentum densities; Eigenvalues; Critical points; Topography; Bond directionality.

\section{Introduction}

Topographical analysis of various scalar fields forms a crucial step in their understanding. Such topographical studies, along with the catastrophe theory, have proved to be useful in the analysis of instabilities observed in different phenomena in the diversified fields of science at large [1]. Within the regime of chemistry also, a number of studies have demonstrated the utility of such an analysis. In the case of electronic structure studies, the topographical analysis of positionspace electron densities initiated by Bader et al. [2] has found wide application. The topographical study of a scalar field essentially deals with the isolation and characterization of critical points (CPs) of it. The CPs $\left\{\boldsymbol{r}_{\mathrm{c}}\right\}$ for a scalar field are defined to be those, at which $\nabla f=0$. A CP is characterized by the corresponding Hessian matrix $H_{i j}=\partial^{2} f /\left.\partial x_{i} \partial x_{j}\right|_{\boldsymbol{r}=\boldsymbol{r}_{\mathrm{c}}}$. A CP is said to be non-degenerate, if all the eigenvalues of the Hessian matrix at that particular point are nonzero (i.e. if $\operatorname{det}\left(H_{i j}\right) \neq 0$ ). Otherwise the CP is said to be degenerate (i.e. at least one of the eigenvalues of the Hessian is zero). The non-degenerate CPs are characterized by

* Presented at the Sagamore X Conference on Charge, Spin and Momentum Densities, Konstanz, Fed. Rep. of Germany, September 1-7, 1991.

Reprint requests to Professor Dr. Shridhar R. Gadre, Department of Chemistry, University of Poona, Pune-411 007, India. an ordered pair $(R, \sigma)$. Here, $R$ is the rank of the Hessian matrix, which is three here, and $\sigma$ is the excess of positive eigenvalues over the negative ones. Thus, there may arise four types of non-degenerate CPs in three-dimensional scalar fields. A $(3,-3)$ is a maximum, $(3,+3)$ a minimum and $(3,-1)$ and $(3,+1)$ are saddles. The non-degenerate $\mathrm{CP}$ is invariably an isolated one; however, the converse is not necessarily true. The classification of isolated degenerate CPs leads to an important subject of science, the catastrophe theory [3]. However, if CPs lie on a line or a curve, they are non-isolated degenerate CPs.

The electron momentum densities (EMDs) have been a topic of research for a long time. It is interesting to note [4] that the distribution under investigation has no direct information about the nuclear sites and has only one center, viz. $\boldsymbol{p}=0$. Also, for a real timereversal-invariant Hamiltonian, the non-degenerate ground-state wavefunction may be taken to be real, whereby the molecular EMD is inversion symmetric, i.e. $\gamma(\boldsymbol{p})=\gamma(-\boldsymbol{p})$ [5]. The first-ever study (see [4] for details) giving directly the signature of bond formation in momentum space, was through observation of a preferred directionality, i.e. anisotropy in the EMD, of diatomic molecules. Subsequently, this was formalized into a principle, known as the bond-directionality (BD) principle. Tanner [6] has recently analyzed the deeper underpinnings of the BD principle, its ramification as well as its limitations. Tanner [6] proposed a 
new BD principle valid for diatomics which states that "In a chemical bond in a bound molecule in its equilibrium geometry, there are values $\boldsymbol{p}_{\mathrm{m}}$ of momentum which are more probable, i.e. which correspond to local maxima of $\gamma(\boldsymbol{p})$. These values are determined by both the geometric and electronic symmetries of the molecule. For momenta $\boldsymbol{p}=\boldsymbol{p}_{\mathrm{m}}+\delta \boldsymbol{p}$ near a maximum, it is more likely that $\delta \boldsymbol{p}$ is perpendicular rather than parallel to the bond axis". Anchell and Harriman [7] have recently investigated the BD principle and anisotropies in molecular EMDs via the Husimi function. Also a generalized BD principle applicable to planar polyatomic molecules was further suggested by them. Thus, the BD principle has been a subject of interest for the past few decades.

Apart from the aforementioned ones, many other investigations on molecular EMDs have helped in its understanding. Studies on difference momentum densities [8], symmetry [4a, 9] and partitioning of the EMD via partial-wave analysis [10] are particularly noteworthy. The application of difference momentum-density maps $\Delta \gamma(\boldsymbol{p})$ is simple only for diatomic molecular systems [8]. For a general, three-dimensional polyatomic molecule, it is rather difficult to extract useful chemical information directly from such $\Delta \gamma(\boldsymbol{p})$-maps. Furthermore, the inversion symmetry of the EMD leads to higher molecular symmetries in momentum space $[4 \mathrm{a}, 9,10]$ as compared to that in coordinate space. Molecular symmetry also plays an important rôle in the partitioning of the molecular EMD [10]. For linear molecules, the molecular EMD can be partitioned using Legendre polynomials as

$$
\gamma(\boldsymbol{p})=\gamma_{0}(p)+\gamma_{2}(p) P_{2}(\cos \theta)+\gamma_{4}(p) P_{4}(\cos \theta)+\ldots
$$

Here, $\gamma_{0}(p)$ is the isotropic momentum density, and all the other terms introduce anisotropies to the total molecular EMD of linear molecules. Thakkar and Pedersen [11] have further partitioned the spherically averaged EMDs in a MacLaurin expansion as

$$
\gamma_{0}(p)=A_{0}+A_{2} p^{2}+A_{4} p^{4}+\ldots
$$

The leading MacLaurin coefficients have been reported for a variety of linear molecules. The criticality at $p=0$ for spherically averaged EMDs was obtained from $A_{2}$ in (2), which will be discussed later.

It is the purpose of the present study to investigate the topography of some linear molecules and to understand its repercussions, in line with the aforementioned studies on the critical structure of molecular EMDs.

\section{Topography of Linear Molecules}

The molecular wavefunctions employed in this study were generated using the program TURBOMOLE [12] with geometry optimization and a triple-zeta basis set [13] for all the molecules. In order to investigate the possible basis-set dependencies of the critical structure, some molecular systems were studied using STO-3G, double-zeta [14] and some polarized basissets.

That $\boldsymbol{p}=0$ is always a critical point in the topology of molecular EMDs [4] is a consequence of inversion symmetry. A further consequence of that fact is that the total number of non-degenerate CPs in the topology of molecular EMDs is always odd. Because of this inversion-symmetric nature of molecular EMDs, linear molecules have $D_{\infty \mathrm{h}}$ symmetry in p-space [10]. The topographical consequence of this symmetry is that, whenever a CP occurs off the $p_{z}$-axis (in all calculations, the $z$-axis is the coordinate-space internuclear axis), it forms a ring of nonisolated degenerate CPs having constant EMD value. It has been pointed out in [4b] that the direction of degeneracy does not remain constant for such a ring. Unlike its $\boldsymbol{r}$-space counterpart $\varrho(\boldsymbol{r})$, this occurrence of a ring of degenerate CPs is an interesting property of the $\boldsymbol{p}$-space density.

In [4b] we have pointed out a definite hierarchy in the occurrence of CPs in the EMD topology of diatomic as well as polyatomic molecules: The occurrence of a minimum at $\boldsymbol{p}=0$ implies the possible existence of all other types of criticalities, viz. $(3,+1)$, $(3,-1)$ and $(3,-3)$, elsewhere in the EMD distribution. The existence of a $(3,+1)$ saddle at $p=0$ shows only $(3,-1)$ and $(3,-3)$-type CPs away from the origin, and for a $(3,-1) \mathrm{CP}$ at the origin, only $(3,-3)$ type CPs occur at $\boldsymbol{p} \neq 0$. If a maximum is exhibited at $\boldsymbol{p}=0$, then the EMD is deprived of any other CP in its topology. Thus it can be remarked that the $\mathrm{CP}$ at $\boldsymbol{p}=0$ is a harbinger of all other CP types in the molecular topology.

It is also known [15] that the number of critical points is related to the Euler characteristic of space by the Pioncare-Hopf $(\mathrm{PH})$ theorem for a three-dimensional scalar field that is smooth and positive everywhere. The relation is

$$
n_{-3}-n_{-1}+n_{+1}-n_{+3}=1,
$$

where $n_{-3}$ and $n_{+3}$ are the numbers of maxima and minima, respectively, whereas $n_{-1}$ and $n_{+1}$ are the 
Table 1. Table of the topography of $\gamma(\boldsymbol{p})$ of some linear molecules using a triple-zeta basis set (all values are in a.u.).

\begin{tabular}{|c|c|c|c|c|c|c|c|}
\hline \multirow[t]{2}{*}{ Molecule } & \multirow[t]{2}{*}{ Energy } & \multirow{2}{*}{$\begin{array}{l}\text { Bond } \\
\text { length }\end{array}$} & \multirow[t]{2}{*}{$\gamma(0)$} & \multicolumn{2}{|c|}{ Eigenvalues of $\mathrm{CP}$ at $\boldsymbol{p}=0$} & \multirow{2}{*}{$\begin{array}{l}\text { Location of CP away } \\
\text { from } p=0\end{array}$} & \multirow{2}{*}{$\begin{array}{l}\text { Type of CP } \\
\text { away from } \\
\boldsymbol{p}=0\end{array}$} \\
\hline & & & & $\lambda_{1}=\lambda_{2}$ & $\lambda_{3}$ & & \\
\hline $\mathrm{AlH}$ & -242.455 & 3.1142 & 4.517 & -53.150 & 19.550 & $(0.0,0.0, \pm 0.18)$ & $(3,-3)$ \\
\hline $\mathrm{OH}^{-}$ & -75.396 & 1.7850 & 0.629 & 3.782 & 2.937 & $\begin{array}{l}(0.0,0.0, \pm 0.46) \\
(0.40,0.30,0.0)\end{array}$ & $\begin{array}{l}(3,+1) \\
\text { Deg } \mathrm{CP}^{\mathrm{a}}\end{array}$ \\
\hline $\mathrm{HF}$ & -100.049 & 1.6973 & 0.368 & 1.126 & 1.075 & $(0.0,0.0, \pm 0.54)$ & $(3,+1)$ \\
\hline $\begin{array}{l}\mathrm{N}_{2} \\
\mathrm{NaH}\end{array}$ & $\begin{array}{l}-108.980 \\
-162.378\end{array}$ & $\begin{array}{l}2.0185 \\
3.6258\end{array}$ & $\begin{array}{l}1.251 \\
5.105\end{array}$ & $\begin{array}{r}0.668 \\
-89.200\end{array}$ & $\begin{array}{l}3.811 \\
-73.360\end{array}$ & $(-0.11,0.18,0.0)$ & Deg. $C P^{a}$ \\
\hline $\mathrm{NaF}$ & -261.358 & 3.6595 & 0.533 & 5.171 & 6.925 & $\begin{array}{l}(0.0,0.0, \pm 0.41) \\
(-0.38,0.20,0.0)\end{array}$ & $\begin{array}{l}(3,-3) \\
\text { Deg. } \mathbf{C P}^{\mathrm{a}}\end{array}$ \\
\hline $\mathrm{NaCl}$ & -621.435 & 4.5304 & 1.295 & 19.798 & 27.196 & $\begin{array}{l}(0.0,0.0, \pm 0.37) \\
(-0.32,0.20,0.0)\end{array}$ & $\begin{array}{l}(3,-3) \\
\text { Deg. } \mathbf{C P}^{\mathbf{a}}\end{array}$ \\
\hline $\mathrm{FCN}^{\mathrm{b}}$ & -191.722 & $\begin{array}{l}2.4605 \\
2.2000\end{array}$ & 1.118 & 6.778 & 3.279 & $\begin{array}{l}(0.23,-0.40,0.0) \\
(0.0,0.0,+0.38)\end{array}$ & $\begin{array}{l}\text { Deg. CP } \\
(3,+1)\end{array}$ \\
\hline $\mathrm{SCN}^{-}$ & -489.930 & $\begin{array}{l}3.1901 \\
2.1605\end{array}$ & 2.218 & 15.711 & 7.750 & $\begin{array}{l}(0.0,0.0, \pm 0.28) \\
(0.25,0.30,0.0)\end{array}$ & $\begin{array}{l}(3,+1) \\
\text { Deg. } C P^{a}\end{array}$ \\
\hline $\begin{array}{l}\mathrm{BH} \\
\mathrm{BF}\end{array}$ & $\begin{array}{l}-25.129 \\
-124.154\end{array}$ & $\begin{array}{l}2.3067 \\
2.3688\end{array}$ & $\begin{array}{l}2.312 \\
2.567\end{array}$ & $\begin{array}{l}-18.457 \\
-19.065\end{array}$ & $\begin{array}{r}8.877 \\
-16.428\end{array}$ & $(0.0,0.0, \pm 0.24)$ & $(3,-3)$ \\
\hline $\mathrm{FCCH}$ & -175.711 & $\begin{array}{l}2.3796 \\
2.2163 \\
1.9894\end{array}$ & 1.487 & 7.970 & -0.258 & $(0.25,0.29,0.0)$ & Deg. $\mathrm{CP}^{\mathrm{a}}$ \\
\hline $\mathrm{CN}^{-}$ & -92.333 & 2.2000 & 2.106 & 1.458 & -9.247 & $(0.12,-0.12,0.0)$ & Deg. $\mathrm{CP}^{\mathrm{a}}$ \\
\hline
\end{tabular}

a point on the ring of nonisolated degenerate critical points.

For FCN the table shows $R(\mathrm{FC})$ and $R(\mathrm{CN})$, for $\mathrm{SCN}^{-}$they are $R(\mathrm{SC})$ and $R(\mathrm{CN})$ and for $\mathrm{FCCH}, R(\mathrm{FC}), R(\mathrm{CC})$, and $R(\mathrm{CH})$ in that order.

numbers of $(3,-1)$ and $(3,+1)$ saddles. The molecular EMD distribution is also smooth and positive throughout and hence satisfies the $\mathrm{PH}$ relation. This relation, in conjunction with the above mentioned hierarchy, restricts the occurrence of the number of CPs of a particular type. This condition can also be used as a check on the occurrence of CPs. For linear molecules having a minimum at $\boldsymbol{p}=0$, a pair of $(3,+1)$ or $(3,-3)$-type CPs is found on the $p_{z}$-axis, symmetric about the origin (cf. Tables 1 and 2). This occurrence of a pair of non-degenerate CPs is in accordance with the $\mathrm{PH}$ relation. In addition to these $\mathrm{CPs}$, a ring or rings of nonisolated degenerate CPs could also be possible. Even though for molecules with a $(3,+1) \mathrm{CP}$ at the origin the existence of equal numbers of $(3,-1)$ and $(3,-3) \mathrm{CPs}$ is allowed elsewhere (owing to the $\mathrm{PH}$ theorem), these systems are observed to be devoid of any type of non-degenerate CP. A ring of nonisolated degenerate CPs is always observed in the $\gamma(\boldsymbol{p})$ topology of these molecules studied here. Further, a molecular $(3,-1) \mathrm{CP}$ at $\boldsymbol{p}=0$ leads to the existence of a pair of maxima on the $p_{z}$-axis, as may be seen for the AlH molecule, illustrated in Table 1. For molecules having a maximum at $\boldsymbol{p}=0$, it is a unique $\mathrm{CP}$ in the topology of the molecule, in conformity with the $\mathrm{PH}$ relation given in (3). Table 1 also gives the EMD values at $\boldsymbol{p}=0$ for different molecular systems and the location as well as the type of CPs away from the origin.

Table 2 shows the basis-set effect for a few linear molecular systems. It can be seen that all basis sets studied yield identical critical structures at $\boldsymbol{p}=0$ for molecules such as $\mathrm{H}_{2}, \mathrm{LiH}, \mathrm{Li}_{2}$, and $\mathrm{CO}$ (these molecules have a unique $\mathrm{CP}$ in their topology, a maximum at the origin). However, for other systems like $\mathrm{C}_{2} \mathrm{H}_{2}, \mathrm{HCN}$, and $\mathrm{HCl}$ it is clearly discernible that a minimal basis is not sufficient to provide the critical structure exhibited by both double-zeta (DZ) and polarized basis sets (e.g. 6-31G** and triple-zeta polarized, TZP). The critical structure exhibited by the $\mathrm{DZ}$ basis is identical with the one of the polarized basis for all the sample molecular systems, implying the adequacy of the DZ basis to bring out the topographical features of the EMD for linear molecules.

It is well known [2] that the Laplacian of a function provides a measure of concentration and depletion of the function. A positive value of the Laplacian implies depletion, whereas a negative value indicates the concentration of momentum density. According to the reciprocity in $\boldsymbol{r}$ - and $\boldsymbol{p}$-space properties, a negative 
Table 2. Table of the topographical information of $\gamma(\boldsymbol{p})$ for some linear molecules for different basis sets (all values are in a.u.).

\begin{tabular}{|c|c|c|c|c|c|c|c|}
\hline \multirow[t]{2}{*}{ Molecule } & \multirow{2}{*}{$\begin{array}{l}\text { Basis set } \\
\text { with } \\
\text { energy }\end{array}$} & \multirow{2}{*}{$\begin{array}{l}\text { Bond } \\
\text { length }\end{array}$} & \multirow[t]{2}{*}{$\gamma(0)$} & \multicolumn{2}{|c|}{ Eigenvalues of $\mathrm{CP}$ at $\boldsymbol{p}=0$} & \multirow{2}{*}{$\begin{array}{l}\text { Location of CP away } \\
\text { from } p=0\end{array}$} & \multirow{2}{*}{$\begin{array}{l}\text { Type of CP } \\
\text { away from } \\
p=0\end{array}$} \\
\hline & & & & $\lambda_{1}=\lambda_{2}$ & $\lambda_{3}$ & & \\
\hline \multirow[t]{3}{*}{$\mathrm{H}_{2}$} & STO-3G & 1.3999 & 0.955 & -4.2721 & -5.1373 & & \\
\hline & $\begin{array}{l}\mathrm{DZ}^{\mathrm{a}} \\
(-1.1266)\end{array}$ & 1.4016 & 1.017 & -4.5592 & -5.5583 & & \\
\hline & $\begin{array}{l}\text { DZP } \\
(-1.1313)\end{array}$ & 1.3872 & 1.103 & -5.4196 & -6.4430 & & \\
\hline \multirow[t]{3}{*}{$\mathrm{LiH}$} & $\begin{array}{l}\text { STO-3G } \\
(-7.8634)\end{array}$ & 2.8616 & 3.279 & -47.716 & -22.843 & & \\
\hline & $\begin{array}{l}3-21 G \\
(-7.9316)\end{array}$ & 3.0875 & 4.462 & -91.821 & -60.411 & & \\
\hline & $\begin{array}{l}\mathrm{DZ} 2 \mathrm{p} \\
(-7.9795)\end{array}$ & 3.0072 & 4.188 & -71.347 & -84.422 & & \\
\hline \multirow[t]{3}{*}{$\mathrm{Li}_{2}$} & $\begin{array}{l}\text { STO-3G } \\
(-14.639)\end{array}$ & 5.0830 & 20.960 & -203.39 & -282.43 & & \\
\hline & $\begin{array}{l}3-21 \mathrm{G} \\
(-14.769)\end{array}$ & 5.2761 & 20.346 & -626.59 & -825.19 & & \\
\hline & $\begin{array}{l}\mathrm{DZ} \mathrm{2p} \\
(-14.868)\end{array}$ & 5.2639 & 19.490 & -545.53 & -787.50 & & \\
\hline \multirow[t]{3}{*}{$\mathrm{CO}$} & $\begin{array}{l}\text { STO-3G } \\
(-111.23)\end{array}$ & 2.1646 & 1.030 & -1.2503 & -1.5770 & & \\
\hline & $\begin{array}{l}\mathrm{DZ} \\
(-112.68)\end{array}$ & 2.1320 & 1.450 & -2.7331 & -4.6590 & & \\
\hline & $\begin{array}{l}6-31 \mathrm{G}^{* *} \\
(-112.74)\end{array}$ & 2.1048 & 1.388 & -2.4744 & -4.0260 & & \\
\hline \multirow[t]{3}{*}{$\mathrm{C}_{2} \mathrm{H}_{2}{ }^{\mathrm{c}}$} & $\begin{array}{l}\text { STO-3G } \\
(-75.856)\end{array}$ & $\begin{array}{l}2.0132 \\
2.2081\end{array}$ & 1.785 & -2.5499 & -4.1947 & & \\
\hline & $\begin{array}{l}\mathrm{DZ} \\
(-76.792)\end{array}$ & $\begin{array}{l}2.0030 \\
2.2734\end{array}$ & 1.772 & 7.1738 & -4.4047 & $(0.18,-0.31,0.0)$ & Deg. $\mathrm{CP}^{\mathrm{b}}$ \\
\hline & $\begin{array}{l}6-31 \mathrm{G}^{* *} \\
(-76.822)\end{array}$ & $\begin{array}{l}1.9968 \\
2.2414\end{array}$ & 1.746 & 2.2978 & -4.4429 & $(-0.01,-0.30,0.0)$ & Deg. $\mathrm{CP}^{\mathrm{b}}$ \\
\hline \multirow[t]{3}{*}{$\mathrm{HCN}^{\mathrm{d}}$} & $\begin{array}{l}\text { STO-3G } \\
(-91.675)\end{array}$ & $\begin{array}{l}2.0213 \\
2.1787\end{array}$ & 1.358 & -1.3652 & -2.9437 & & \\
\hline & $\begin{array}{l}\mathrm{DZ} \\
(-92.829)\end{array}$ & $\begin{array}{l}2.0091 \\
2.1833\end{array}$ & 1.438 & 3.4957 & -2.2652 & $(-0.19,0.29,0.0)$ & Deg. $\mathrm{CP}^{\mathrm{b}}$ \\
\hline & $\begin{array}{l}6.31 \mathrm{G}^{* *} \\
(-92.877)\end{array}$ & $\begin{array}{l}2.0096 \\
2.1405\end{array}$ & 1.465 & 1.0438 & -3.7899 & $(-0.04,-0.26,0.0)$ & Deg. CP ${ }^{b}$ \\
\hline \multirow[t]{2}{*}{$\mathrm{HCl}$} & $\begin{array}{l}\text { STO-3G } \\
(-455.16)\end{array}$ & 2.4976 & 1.184 & -4.5925 & 2.8898 & $(0.0,0.0, \pm 0.35)$ & $(3,-3)$ \\
\hline & $\begin{array}{l}\text { TZP } \\
(-460.09)\end{array}$ & 2.5000 & 1.014 & 7.4593 & 5.9596 & $\begin{array}{l}(-0.33,-0.30,0.0) \\
(0.0,0.0, \pm 0.41)\end{array}$ & $\begin{array}{l}\text { Deg. CP }{ }^{b} \\
(3,+1)\end{array}$ \\
\hline
\end{tabular}

${ }^{a}$ DZ basis stands for geometry and basis taken from Snyder and Basch [14]. ${ }^{\mathrm{b}} \mathrm{A}$ point on the ring of nonisolated degenerate critical points. $-{ }^{\mathrm{c}}$ The first bond length indicates $R(\mathrm{HC})$, the second one $R(\mathrm{CC}) .{ }^{\mathrm{d}}$ The first bond length indicates $R(\mathrm{HC})$, the second one $R(\mathrm{CN})$.

Laplacian of the EMD at $\boldsymbol{p}=0$ may imply that the charge density in $\boldsymbol{r}$-space will be localized with only a small charge outside a certain finite boundary, i.e. in the region of larger $|\boldsymbol{r}|$. Similarly, a positive Laplacian of the EMD at the origin implies a relatively large charge outside a certain finite boundary. The studies of exterior electron densities (EED) by Ohno and Ishida [16] reveal that the higher the charge in the wavefunction tail, the better is the quality of the wavefunction. Thus, depletion of the momentum density at $\boldsymbol{p}=0$ implies the possibility of a higher charge density in the exterior region of the van der Waals surface, i.e. a higher EED, in accordance with the Fourier-transformation principle. As can be seen from Table 2, DZ wavefunctions, in general, have higher depletion (i.e. a higher positive Laplacian of the EMD) and hence have a better wavefunction tail. Minimal basis sets always exhibit a poor behaviour in the exterior region, which is in agreement with the studies by Ohno and Ishida [16].

As remarked by several authors $[6,17]$, a reliable method of describing the BD principle in a quantita- 
tive way that also takes into account the cognizance of the BD away from the origin, is necessary. In the following part, we investigate the consequences of the BD principle for the topography of molecular EMDs with a view to gain knowledge of the principle in depth.

In [ $4 \mathrm{~b}$ ] we have suggested that the curvature (i.e. eigenvalues) of a CP at $\boldsymbol{p}=0$ can be looked upon as a measure of anisotropies in molecular EMDs. Here, we propose the use of similar topographical considerations for studies of the BD in linear molecules. The curvatures of the most crucial and inevitable $\mathrm{CP}$ in the topology of a molecular EMD, viz. the one at $\boldsymbol{p}=0$, can be looked upon as just one of the measures of the $\mathrm{BD}$ in diatomic molecules. The higher the magnitude of curvature along any momentum direction, the greater is the increase or falloff of the EMD, depending on whether the sign of curvature is positive or negative, respectively. For linear molecules, the Hessian matrix at $\boldsymbol{p}=0$ is diagonal and hence the curvatures $\lambda_{1}$ and $\lambda_{2}$ correspond to the $p_{x}$ and $p_{y}$-directions, respectively. Thus, the magnitudes of curvatures suggest the quantification of the BD in diatomic molecules. For the $\mathrm{H}_{2}$ molecule (cf. Table 2), these eigenvalues imply that the relative change in the EMD is greater in the $p_{z}$-direction as compared to perpendicular directions, supporting the well-known BD principle. A similar consideration, applied to the other molecules in Tables 1 and 2 reveals that there are a few molecular systems like $\mathrm{AlH}, \mathrm{NaH}, \mathrm{OH}^{-}$and HF (for the TZP basis) that do not obey the said BD principle in terms of the above quantification. It is also known from earlier studies [7] that ionic molecules such as $\mathrm{AlH}$ and $\mathrm{NaH}$ do not obey the $\mathrm{BD}$ principle. However, for $\mathrm{OH}^{-}$and $\mathrm{HF}$ it is important to note that they have a minimum $\boldsymbol{p}=0$. The $\mathrm{PH}$ relation induces the EMD topology to have CPs of the kind $(3,+1)$ on the $p_{z}$-axis, Also, as pointed out earlier [6], few molecular systems show elongated contours away from the origin. This elongation must be about a pair of $(3,+1)$ CPs on the $p_{z}$-axis. Thus, a modified approach towards BD is the following: all non-degenerate CPs on the bonding axis should be considered for a measure of $\mathrm{BD}$. The investigation of eigenvalues of the CP on the bond axis (away from $p=0$ ) for $\mathrm{HF}$ and $\mathrm{OH}^{-}$shows that the relative change of the EMD along the $p_{z}$-axis at these CPs is greater than the one in the corresponding perpendicular directions. Molecules with $(3,+1)$ and $(3,-3) \mathrm{CPs}$ at $\boldsymbol{p}=0$ are deprived of any other type of non-degenerate CP for $\boldsymbol{p} \neq 0$. Hence for such molecular systems only the curvatures of the CP at $\boldsymbol{p}=0$ are considered. For the $\mathrm{NaF}$ molecule, the BD principle seems to be valid at $\boldsymbol{p}=0$ and even for CPs on the $p_{z}$-axis, whereas for $\mathrm{BH}$ it is not valid at all with three CPs in its topology. Thus, a molecular system will be said to satisfy the BD principle only if that principle is seen to be obeyed by all non-degenerate CPs of the EMD. This new approach to the BD principle gives rise to a broad classification of all linear molecules into three distinct groups, viz. those which obey the BD principle fully, i.e. by all non-degenerate CPs in its topology (e.g. $\mathrm{N}_{2}$ and $\mathrm{CN}^{-}$ in Table 1), those which do not obey it at all (e.g. BH, $\mathrm{BF}$ and $\mathrm{NaH}$ in Table 1), and a new class of molecules that obey it partially (e.g. $\mathrm{OH}^{-}$and $\mathrm{HF}$ in Table 1).

Another well-studied characteristic of molecular EMDs is the partitioning of the molecular EMD [10, 11], which will be investigated hereafter from a topographical point of view. Thakkar and Pedersen [11] have studied the critical structure of the spherically averaged EMD, $\gamma_{0}(p)$ at $p=0$. The coefficient $A_{2}$ of the MacLaurin series (see (2)) decides the critical structure of $\gamma_{0}(p)$ : if $A_{2}$ is positive for a molecule, the corresponding $\gamma_{0}(p)$ has a minimum at $p=0$, whereas negative $A_{2}$ implies a maximum at $p=0$. It is indeed interesting to note that the critical structure exhibited by the spherically averaged EMD at $p=0$ is generally retained in the full three-dimensional molecular EMD at $\boldsymbol{p}=0$. A comparison of Table I of [11] with Tables 1 and 2 of the present study reveals that molecules having a maximum or minimum at $\boldsymbol{p}=0$ exactly match the criticality at $p=0$ for $\gamma_{0}(p)$. However, saddles in a molecular EMD require a different viewpoint for the comparison of criticality of $\gamma_{0}(p)$. Thus, one can infer that the critical structure of the molecular EMD at $\boldsymbol{p}=0$ is largely dictated by the criticality of $\gamma_{0}(p)$ at $p=0 . \gamma_{0}(p)$ being the leading term in the expansion of $\gamma_{0}(\boldsymbol{p})$, as given in (1) for linear molecules, the above behaviour is heuristically expected.

\section{Summary}

In the present study, a detailed topographical analysis of the electron momentum densities of some linear molecules is presented. The curvatures of the CP at $\boldsymbol{p}=0$ and elsewhere on the bond axis decide the BD for a molecule. The topographical analysis is found to be useful in the quantification of the BD principle and also takes cognizance of elongation of $\gamma(\boldsymbol{p})$ contours away from the origin. In fact, all linear molecules can broadly 
be classified into three types: i) those which totally conform to the $\mathrm{BD}$, ii) those which do not obey the $\mathrm{BD}$ at all and iii) those which partially satisfy the BD. Also, the repercussions of the hierarchy of CPs in molecular EMDs in conjunction with the Poincaré-Hopf theorem lead to a definite critical structure for linear molecules. Further, it has been pointed out that for many linear molecules the critical structure at $\boldsymbol{p}=0$ is largely dominated by the CP of the spherically averaged EMD at $p=0$. Thus we hope to convey the message that topographical analysis is an important tool for the understanding of molecular EMDs.

[1] T. Poston and I. Stewart, Catastrophe Theory and its Applications, Pitman, London 1978. - P. T. Saunders, An Introduction to Catastrophe Theory, Cambridge University Press, Cambridge 1980. - T. Poston and I. Stewart, Taylor Expansions and Catastrophes, Research Notes in Mathematics 7, Pitman, London 1976.

[2] R. F. W. Bader, Atoms in Molecules: A Quantum Theory, Oxford University Press, Oxford 1990, and references therein.

[3] M. V. Berry, Adv. Phys. 25, 1 (1976). - M. V. Berry and M. R. Mackloy, Philos. Trans. Roy. Soc. London 287, 1 (1977). - E. C. Zeeman, Sci. Amer. 234, 65 (1976). I. Stewart, Sci. Amer. 264 (6), 123 (1991).

[4a] S. R. Gadre, A. C. Limaye, and S. A. Kulkarni, J. Chem. Phys. 94, 8040 (1991).

[4 b] S. A. Kulkarni, S. R. Gadre, and R. K. Pathak, Phys. Rev. A 45, 4399 (1992).

[5] P.-O. Löwdin, Adv. Quantum Chem. 3, 323 (1967). P. Kaijser and V. H. Smith, Jr., in: Quantum Science (J. L. Calais, O. Goscinski, J. Linderberg, and Y. Öhrn, eds.), Plenum, New York 1976.

[6] A. C. Tanner, Chem. Phys. 123, 241 (1988).

[7] J. L. Anchell and J. E. Harriman, J. Chem. Phys. 89, 6860 (1988). - J. A. Anchell and J. E. Harriman, J. Chem. Phys. 92, 2943 (1990).
Acknowledgements

S.A.K. thanks the Council of Scientific and Industrial Research (CSIR), New Delhi for providing a grant to attend the Sagamore X Conference. S.R.G. thanks the Alexander von Humboldt-Stiftung for the award of a Research Fellowship. We thank Professor Reinhart Ahlrichs for providing work facilities. Thanks are also due to Sangeeta Bapat and A. Taspa for computational assistance.

[8] W. H. Henneker and P. E. Cade, Chem. Phys. Letters 2, 575 (1968). - A. Rozendaal and E. J. Baerends, Chem. Phys. 95, 57 (1985). - B. I. Ramirez, J. Phys. B: At. Mol. Phys. 15, 4339 (1982) and references therein.

[9] M. Defranceschi and G. Berthier, J. Phys. France 51, 2791 (1990).

[10] A. J. Thakkar, A. M. Simas and V. H. Smith, Jr., J. Chem. Phys. 81, 2953 (1984). - E. Heuser-Hofmann and W. Weyrich, Z. Naturforsch. 40 a, 99 (1985).

[11] A. J. Thakkar and W. A. Pedersen, Intern. J. Quantum Chem. S 23, 245 (1989).

[12] The package TURBOMOLE developed by R. Ahlrichs and co-workers at University of Karlsruhe, Germany. See R. Ahlrichs, M. Bär, M. Häser, H. Horn, and C. Kölmel, Chem. Phys. Letters 162, 165 (1990).

[13] $(10 \mathrm{~s}, 6 \mathrm{p}, 1 \mathrm{~d})$ basis from the HONDO basis-set library with Dunning contraction $(10 \mathrm{~s} / 5 \mathrm{~s}, 6 \mathrm{p} / 3 \mathrm{p}, 1 \mathrm{~d} / 1 \mathrm{~d})$.

[14] L. C. Snyder and H. Basch, Molecular wave functions and properties, Wiley, New York 1972.

[15] K. Collard and G. G. Hall, Intern. J. Quantum Chem. 12, 623 (1977).

[16] K. Ohno and T. Ishida, Intern. J. Quantum Chem. 29, 677 (1986) and references therein.

[17] C. E. Brion, J. P. D. Cook, I. G. Fuss, and E. Weigold, Chem. Phys. 64, 287 (1982). 